\title{
Statistical analysis of Stromboli VLP tremor in the band [0.1-0.5] Hz: some consequences for vibrating structures
}

\author{
E. De Lauro ${ }^{1}$, S. De Martino ${ }^{1,2,3}$, M. Falanga ${ }^{1,2,3}$, and M. Palo ${ }^{1}$ \\ ${ }^{1}$ Dipartimento di Fisica, Salerno University, via S. Allende, I-84081 Baronissi (SA), Italy \\ ${ }^{2}$ INFM, unità di Salerno, via S. Allende, I-84081 Baronissi (SA), Italy \\ ${ }^{3}$ INFN, gruppo collegato di Salerno, via S. Allende, I-84081 Baronissi (SA), Italy
}

Received: 16 January 2006 - Revised: 24 May 2006 - Accepted: 1 June 2006 - Published: 10 August 2006

\begin{abstract}
We analyze time series of Strombolian volcanic tremor, focusing our attention on the frequency band [0.1$0.5] \mathrm{Hz}$ (very long period (VLP) tremor). Although this frequency band is largely affected by noise, we evidence two significant components by using Independent Component Analysis with the frequencies, respectively, of $\sim 0.2$ and $\sim 0.4 \mathrm{~Hz}$. We show that these components display wavefield features similar to those of the high frequency Strombolian signals $(>0.5 \mathrm{~Hz})$. In fact, they are radially polarised and located within the crater area. This characterization is lost when an enhancement of energy appears. In this case, the presence of microseismic noise becomes relevant. Investigating the entire large data set available, we determine how microseismic noise influences the signals. We ascribe the microseismic noise source to Scirocco wind. Moreover, our analysis allows one to evidence that the Strombolian conduit vibrates like the asymmetric cavity associated with musical instruments generating self-sustained tones.
\end{abstract}

\section{Introduction}

The Stromboli volcano is situated on the homonymous island, in the Eolie Archipelago, not far from the coast of Sicily. The activity of this volcano has been attracting the attention of the geophysical community for long time. It is characterized by a continuous tremor on which frequent explosive events are superimposed and it is present on many volcanoes worldwide. It is known as "Strombolian activity". The seismic wavefield of the Stromboli volcano has been investigated, focusing the attention on the explosion quakes (Chouet et al., 1997, 1998; Del Pezzo et al., 1992; Acernese et al., 2003, 2004; Bottiglieri et al., 2005).

Correspondence to: S. De Martino

(demartino@sa.infn.it)
The properties of the volcanic tremor field are not yet well established. Due to the low signal-to-noise ratio, one is required to perform the analysis by considering a large data set, in order to obtain information on a statistical basis.

Strombolian volcanic tremor and explosion quakes show similar features at high frequency $(>0.5 \mathrm{~Hz})$, both in time and frequency domain (Ciaramella et al., 2004; De Lauro, $2006^{1}$ ). This suggests the existence of a common source to be ascribed to the degassing process. Recently, significant components of the volcanic tremor have been evidenced at low frequencies $(<0.5 \mathrm{~Hz})$ (De Martino et al., 2005; De Lauro et al., 2005). One of these components was seen in the frequency band $[0.1-0.5] \mathrm{Hz}$. It displays the same field properties of the high frequency band $(>0.5 \mathrm{~Hz})$, i.e. a radially polarised wavefield and an apparent velocity of $\sim 1 \mathrm{~km} / \mathrm{s}$.

We remind the reader that this frequency band is affected by microseismic noise, whose nature has been investigated for a long time. It is the result of a nontrivial combination of atmospherical processes (e.g. wind waves) and sea waves. The microseismic noise is mainly composed of Raleigh waves. Its most significant spectral peak is around $0.2 \mathrm{~Hz}$ but it can corrupt seismic signals over almost any frequency (e.g. Webb, 1998, and references therein).

This paper is devoted to the careful analysis of this frequency band in order to obtain information about the properties of volcanic tremor and microseismic noise. We analyse the $[0.1-0.5] \mathrm{Hz}$ frequency band (VLP tremor) to infer the possibility of simpler hidden structures.

\footnotetext{
${ }^{1}$ De Lauro, E., De Martino, S., Del Pezzo, E., Falanga, M., Palo, M., and Scarpa, R.: Model for high frequency Strombolian tremor inferred by wavefield decomposition and reconstruction of asymptotic dynamics, J. Geophys. Res., submitted, 2006.
} 


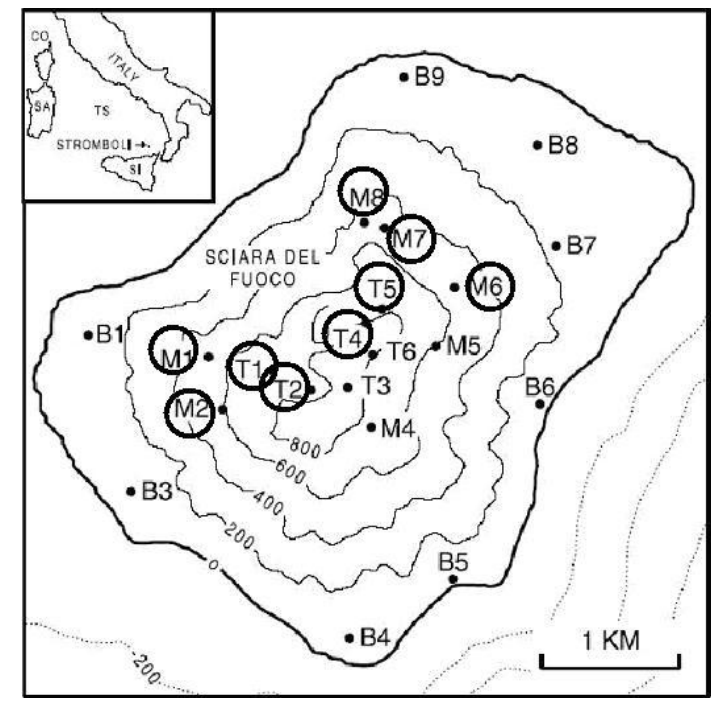

Fig. 1. Location map of the seismic stations. We have analysed the recordings relative to the stations indicated with a circle.

Table 1. Scheme of the operating seismometers selected for our analysis. The effective acquisition time periods for each station are indicated with the symbol $\checkmark$.

\begin{tabular}{ccccc}
\hline & 1st period: & 2nd period & 3rd period & 4th period \\
from & 18 Sep 22:00 UT & 19 Sep 22:00 UT & 22 Sep 11:00 UT & 24 Sep 12:00 UT \\
to & 19 Sep 06:00 UT & 20 Sep 06:00 UT & 23 Sep 12:00 UT & 25 Sep 10:00 UT \\
\hline T1 & $\checkmark$ & $\checkmark$ & $\checkmark$ & $\checkmark$ \\
T2 & $\checkmark$ & $\checkmark$ & $\checkmark$ & $\checkmark$ \\
T4 & $\checkmark$ & $\checkmark$ & $\checkmark$ & $\checkmark$ \\
T5 & NO & NO & $\checkmark$ & $\checkmark$ \\
M1 & $\checkmark$ & $\checkmark$ & $\checkmark$ & $\checkmark$ \\
M2 & $\checkmark$ & $\checkmark$ & $\checkmark$ & $\checkmark$ \\
M6 & $\checkmark$ & $\checkmark$ & NO & $\checkmark$ \\
M7 & NO & $\checkmark$ & NO & $\checkmark$ \\
M8 & $\checkmark$ & $\checkmark$ & $\checkmark$ & NO \\
\hline
\end{tabular}

\section{Data set and preliminary analysis: time energy evolu- tion}

Here, we analyse the large data set recorded during the experiment performed in 1997 (see Chouet et al. (1999, 2003) and references therein). Twenty-one three-component $\mathrm{Gu}-$ ralp CMG-40T broad-band (0.02-60 s) seismometers were deployed on the Stromboli volcano by the U.S. Geological Survey, University of L'Aquila, Osservatorio Vesuviano and National Seismic Survey. The seimometers were set on the flanks of the volcano, forming three rings around the crater area at different altitudes (top, middle and bottom ring indicated on the map with the letters T, M, B, respectively). The distances of the stations from the crater zone ranged from 0.3 to $2.2 \mathrm{~km}$. In Fig. 1, we show the location map of the seismic network.

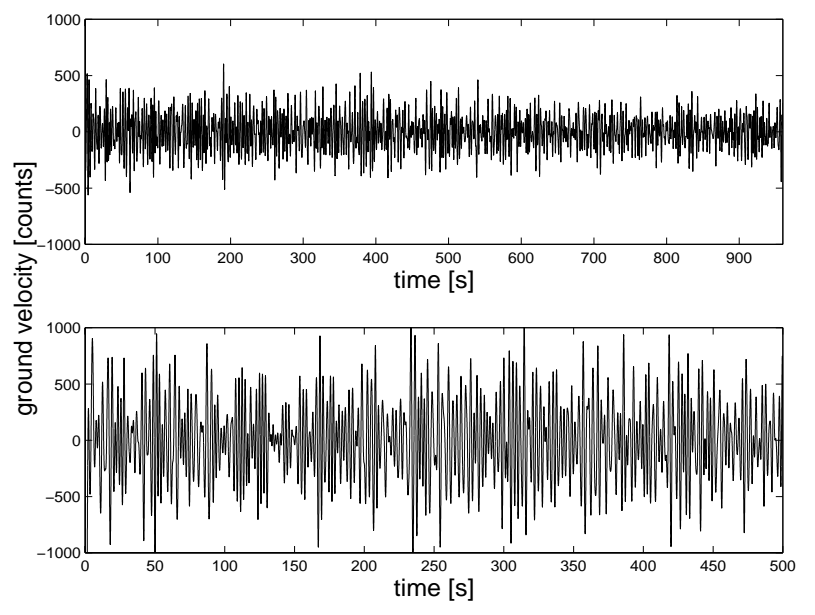

Fig. 2. VLP tremor series recorded at the T1 station, vertical channel. These signals are representative of a tremor (a) recorded on 19 September at 00:33 UT; (b) recorded on 24 September at 12:17 UT. We will see in Sect. 5 that they are characterized by radial and elliptical polarisation, respectively.

The stations have been recording for a week. The whole data set is composed of $60 \mathrm{~h}$ of recorded signals distributed in four disjointed time periods, lasting 7, 7, 25, $21 \mathrm{~h}$, respectively. During these periods not all the instruments have been working.

We have selected for the analysis the best set for our aim. This is composed of the recorded signals along the four time periods indicated above and relative to the nine stations reported in Table 1. As an example of the tremor recorded in different periods, we report in Fig. 2 two representative series of VLP Strombolian tremor.

We start to characterize these four time periods in terms of energy. We evaluate a quantity proportional to the energy by taking the integral of power spectrum. We use for this evaluation sliding windows of $90 \mathrm{~s}$ with an overlapping of $90 \%$. In Fig. 3, we report these evaluated quantities for the four considered time periods by looking at the radial component of the station M2. The other stations display the same behaviour. Notice that the spikes correspond to the explosions; we concentrate our attention on the background energy.

In the first two periods the background energy is almost stationary. In the third period, we observe an enhancement of this background energy followed by a gradual decrease up to a slightly higher cutoff occurring in the fourth period.

We will see that this time evolution of background energy is related to the level of microseismic noise. Accordingly, the polarisation features of the tremor in this frequency band $[0.1-0.5] \mathrm{Hz}$ will vary. 


\section{Independent component analysis}

The first step is to understand what is changing in terms of the sources corresponding to the observed variations of background energy. To achieve this goal we apply Independent Component Analysis (ICA) (for details see Hyvärinen et al., 2001).

It is convenient to give some remarks about this technique (for its application to seismic signal see Acernese et al., 2003).

We can suppose to have $m$ different recorded time series $\boldsymbol{x}$, that we hypothesize to be the linear superposition of $n$ mutually independent unknown sources $\boldsymbol{s}$, due to different mixing, represented by a constant unknown $m \times n$ matrix $\mathbf{A}$. This mixing is essentially due to path, noise, instrumental transfer-functions, etc. If the mixing has to be linear, nothing is assumed with respect to the sources, which can be linear or nonlinear.

Formally, the mixing model is written as

$\boldsymbol{x}=\mathbf{A} \boldsymbol{s}$.

In addition to the source independence request, ICA assumes that the number of available different mixtures $m$ is at least as large as the number of sources $n$. Under these hypothesis, the ICA goal is to obtain a separating matrix $\mathbf{W}$, the inverse of $\mathbf{A}$, in such a way that the vector

$\boldsymbol{y}=\mathrm{W} \boldsymbol{x}$

is an estimate $\boldsymbol{y} \simeq \boldsymbol{s}$ of the original independent source signals.

ICA is a statistical model based on the central limit theorem, which establishes that the distribution of a sum of independent random variables tends towards a Gaussian distribution, under certain conditions. Hence, the main idea of the ICA model is to maximize the non-Gaussianity (superGaussianity or sub-Gaussianity) to extract the independent components. Some heuristic approaches have been proposed in literature to achieve the separation. Among them, a good measure of independence is given by negentropy $J$. It relays upon the information-theoretic quantity of differential entropy $H$ of a random vector and it is defined as follows:

$J(z)=H\left(z_{\text {gauss }}\right)-H(z)$,

where $z$ is a random variable and $z_{\text {gauss }}$ is a Gaussian random variable with the same covariance matrix as $z$. The estimate of negentropy is difficult and, in practice, some approximations must be introduced. These approximations involve the estimate of 4 th order statistical moments.

In the following we shall use the fixed-point algorithm, namely FastICA, based on an approximative Newton iteration scheme. An extensive description of theoretical structure and FastICA algorithm can be found in Hyvärinen et al. (2001).

The basic ICA model, in summary, requires the following assumptions:

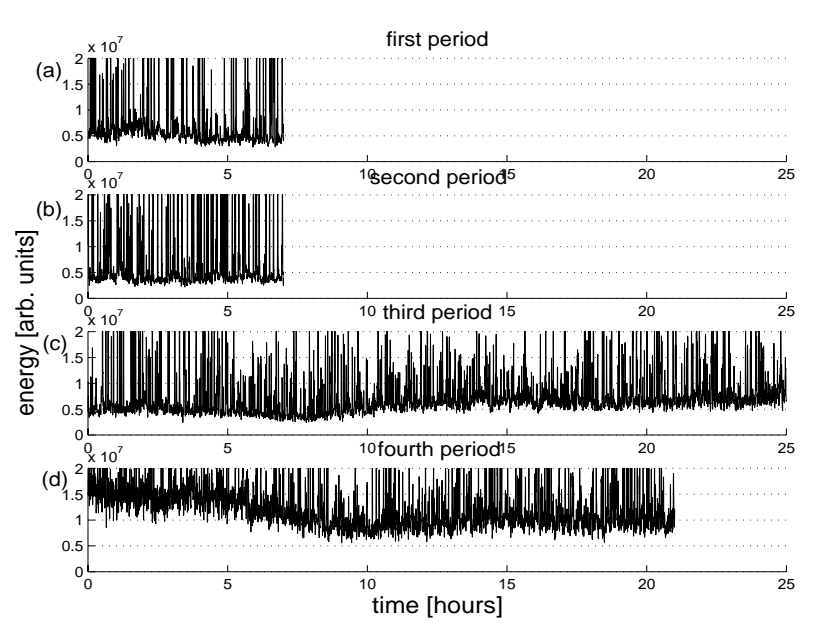

Fig. 3. Time energy evolution estimated for the recordings relative to the M2 station, looking at the four acquisition periods (see Table 1). The spikes correspond to the explosion quakes. The underlying trend is clearly visible (background energy).

- all the independent components, with the possible exception of one component, must be non-Gaussian (Bell and Sejnowski, 1995);

- the number of the observed linear mixtures must be at least as large as the number of independent components;

- the matrix A must be of full column rank.

We have to underline that we cannot recover the amplitudes of the extracted source signals by ICA. In fact, this method cannot determine the variances (proportional to energies) of the extracted independent components, namely little information is assumed on matrix $\mathbf{A}$, therefore, the magnitudes of the basis vectors of the matrix $\mathbf{A}$ and the amplitudes of the source signals can be interchanged in Eq. (1). Therefore, the most natural way to obtain a unique expansion is to assume that each source has a unit variance. This is accomplished by Principal Component Analysis (PCA). This method involves a mathematical procedure that transforms a number of (possibly) correlated variables into a (smaller) number of uncorrelated variables called principal components. The first principal component accounts for as much of the variability in the data as possible, and each succeeding component accounts for the remaining variability. Traditionally, is PCA accomplished by diagonalization of covariance matrix (Bishop, 1995).

\subsection{Application of ICA to VLP tremor}

We have applied ICA to the VLP tremors (considering all available stations) recorded during the first and fourth acquisition periods which were representative of the two observed states: low and high level of energy. We report the results for the recording relative to the vertical direction but the same holds for the other. 

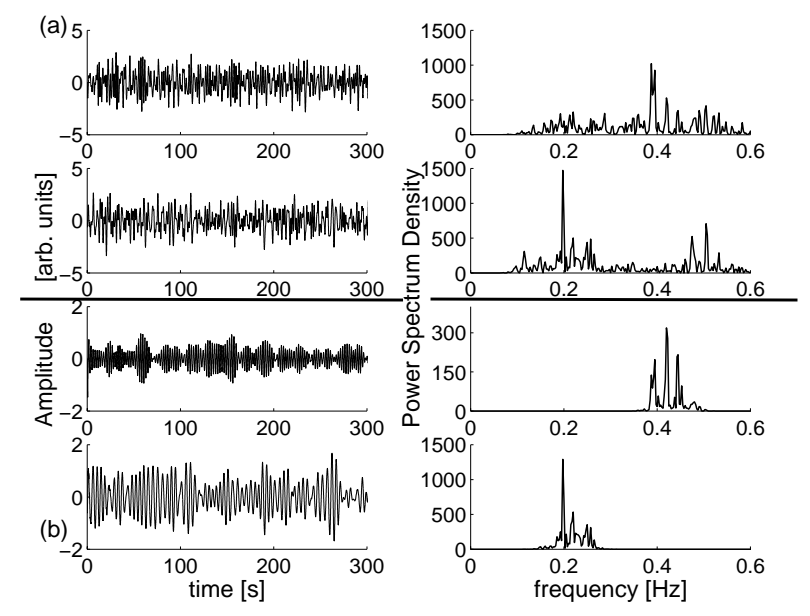

Fig. 4. (a) Components extracted by ICA analyzing the first acquisition period (tremor on 19 September at 00:33 UT, vertical channel recorded at the all stations working in that period). These are the components associated with the principal eigenvalues of the covariance matrix. The relative spectra are reported on the right; (b) the independent components after the application of a nonlinear, denoising method to better evidence the "beats".
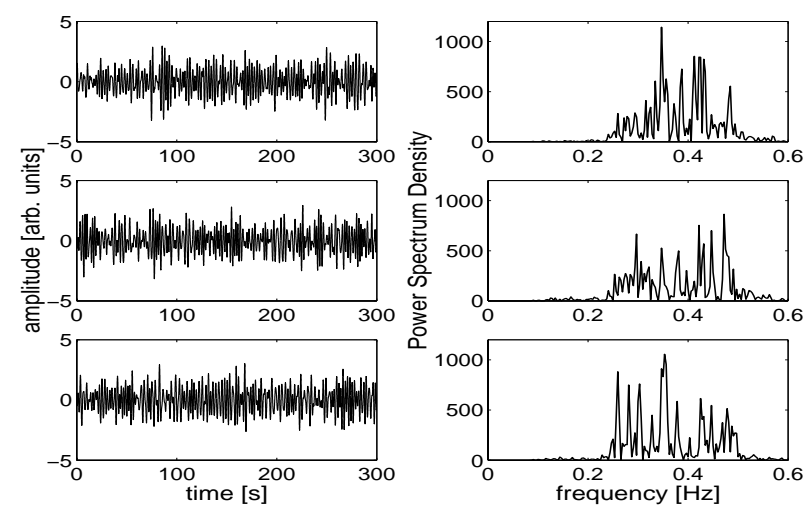

Fig. 5. Components extracted by ICA analyzing the fourth acquisition period (tremor 24 September 12:17 UT, vertical channel, recorded at all stations working in that period). The waveforms and the relative spectra suggest that ICA is not able to isolate independent components.

In the first period, the method has extracted only two independent components with well shaped waveforms and spectral content. These two components are associated with the principal eigenvalues of the covariance matrix. In Fig. 4, we show the extracted waveforms and the corresponding spectra before and after the application of a denoising technique (Kostelich and Schreiber, 1993). The time evolution of both components appears to be characterized by "beats"; this is a common feature of the Strombolian high-frequency wavefield. We may conclude that the low energy state is the linear superposition of two signals peaked, respectively, around 0.2 and $0.4 \mathrm{~Hz}$.
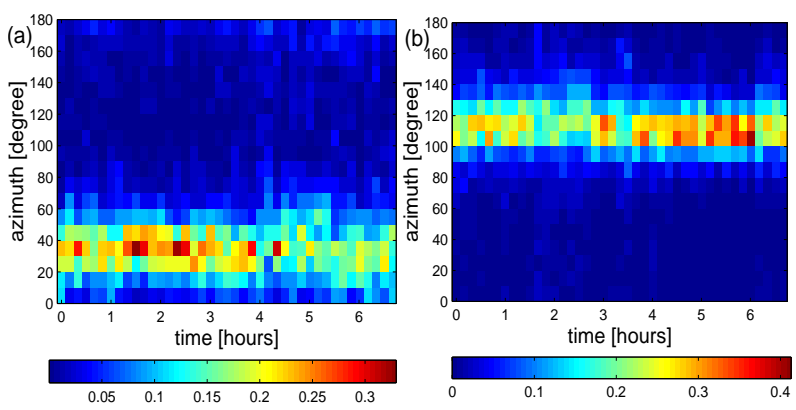

Fig. 6. Example of the evolution of the azimuths during the first (a) and second (b) acquisition periods at stations M2 and T4, respectively: the tremor is radially polarised.

The application of ICA to VLP tremor segments belonging to the first hours of the fourth acquisition period is inefficacious, namely, as one can see in Fig. 5, the previous two modes can still be distinguished in the spectrum (notice that the frequencies are slightly higher than the first case), but they are entangled in an inseparable way. This suggests that the high level of energy may be due to the presence of an external source (microseismic noise), which, superimposing on volcanic source signals, entangles them by a strong coupling (in this case the signals are inseparable).

\section{Polarisation analysis}

To obtain information about the polarisation properties of the tremor, we apply a Kanasewich filter (Kanasewich, 1981). The basic idea of this method is the diagonalization of a covariance matrix computed by the three components of ground motion. The parameters of polarisation (azimuth and dip) are defined in terms of the eigenvector with the highest eigenvalue. Using the eigenvalues of this matrix, we can evaluate a rectilinearity coefficient:

$R L=1-\frac{\lambda_{\min }+\lambda_{\text {int }}}{2 \lambda_{\max }}$

where $\lambda_{\min }, \lambda_{\text {int }}$ and $\lambda_{\max }$ are, respectively, the minimum, intermediate and maximum eigenvalues. If $R L$ is close to one, the waves are linearly polarised and the eigenvector corresponding to the highest eigenvalue defines the direction of polarisation.

We consider the four acquisition periods separately at all the selected stations in order to perform the analysis on a statistical basis. Due to the low signal-to-noise ratio, we look at the results of the polarisation analysis, focusing the attention on the distributions of the three parameters: azimuth, dip and $R L$.

In detail, we apply a Kanasewich filter by considering the frequency band $[0.1-0.5] \mathrm{Hz}$ (excluding the explosions) on windows $5 \mathrm{~s}$ in length, sliding $1 \mathrm{~s}$ every step. We select only 

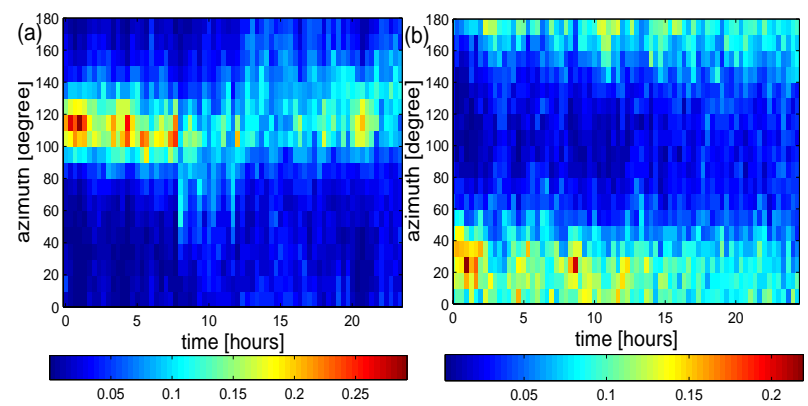

Fig. 7. Example of the evolution of the azimuths relative to the third acquisition period at stations T4 and M2, placed, respectively, one on the east (a) and on the west (b) flank. The behaviour appears to be linked to the energy: when the energy increases, at the east side the azimuths become more dispersive. At the west side the radial direction of the azimuths generally is preserved.

those windows for which $R L$ is high ( $>0.7$ ). Then, we show, as a function of time and azimuth, the fraction of these windows which have every ten minutes azimuths within ten degrees; the color bar (from blue to red) indicates the values assumed by these fractions (see Figs. 6-8).

Figure 6 refers to the first two acquisition periods, each one lasting seven hours and recorded over two different days. The bold red band indicates azimuths pointing towards the crater zone. This feature is common to all the stations. This confirms and generalizes the results of De Lauro et al. (2005).

The third acquisition period $(\sim 25 \mathrm{~h})$ shows a more complex behaviour of azimuths. In Fig. 7, the results of the two stations placed on both flanks of volcano are reported. On the right (Fig. 7b) there are the results relative to the west flank. This graph shows that the polarisation is radial, during the first $11 \mathrm{~h}$, otherwise in the subsequent times, the azimuths become more dispersive. During this period, looking at the evolution of energy, immediately one realizes that polarisation is radiality lost when energy grows. A strong microseismic noise affects the signals. In Fig. 7a, a station in the east flank shows again a radial polarisation in the first hours; then, the azimuth around 120 deg becomes slightly more dispersive.

The behaviour of polarisation at the east flank lets us suppose that the microseismic noise comes from this direction. This is the direction of the Scirocco wind (south, southeast direction) that blows on Stromboli during summer season.

The influence of a source coming from the southeast direction on azimuths is also evident in the fourth acquisition period (Fig. 8). At all the stations a direction of polarisation of 120 deg during the first $6 \mathrm{~h}$ is visible when the energy is high. Then the energy decreases to a level up to its mean value in the third acquisition period, and the azimuths relative to the stations on the east flank show no appreciable variation (see Fig. 8a). The polarisation at stations on the west flank appears more complex (Fig. 8b), namely the distribution of the

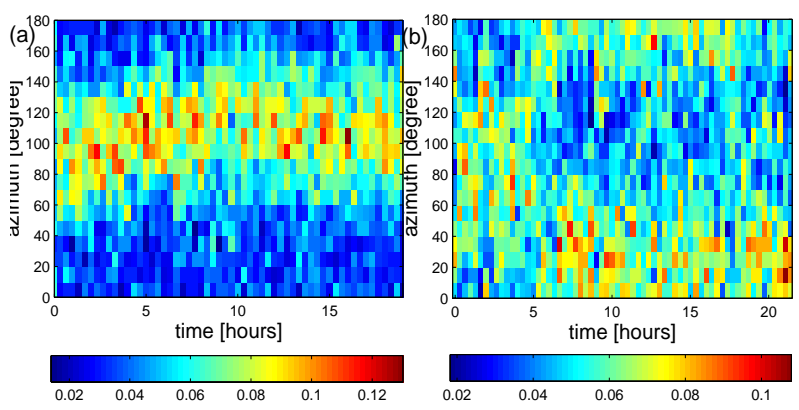

Fig. 8. Example of the evolution of the azimuths during the fourth acquisition period at M7 (a) and T1 (b): the two sides of the volcanic cone manifest different behaviour. When the energy decreases the radial component of the polarisation at the stations on the west flank (see the graph b) become more evident; at the other flank the energy step does not influence the azimuths (see graph a).

azimuths shows two large peaks: one at $\sim 120 \mathrm{deg}$ and the other pointing towards the crater.

Now, we take into account only the period in which polarisation is clearly radial. On the basis of the results of ICA, we subdivide the $[0.1-0.5] \mathrm{Hz}$ frequency band into two bands, respectively, $[0.1-0.3] \mathrm{Hz}$, and $[0.3-0.5] \mathrm{Hz}$ and we apply the polarisation analysis. The window selected for the analysis is $5 \mathrm{~s}$ in length and moves $1 \mathrm{~s}$ every step.

In Fig. 9 the distributions of $R L$, the azimuth and the dip parameters are shown relative to a tremor lasting $6 \mathrm{~min}$. We report only the values of the azimuths and the dip corresponding to a very high $R L(>0.9)$. It appears clear that in these two bands, the wavefield shows radial polarisation. The distributions of the dip parameters indicate little differences in the depth of the source, suggesting the existence of slightly different vibrating structures. Moreover, looking at the $R L$ distribution, the tremor around $0.4 \mathrm{~Hz}$ is better polarised.

The signals recorded at the other stations reveal similar features, but with the influence of their different elevation. For example, at middle stations, dip parameters are slightly more superficial than at the top; the azimuths estimated on the tremor in the band $[0.1-0.3] \mathrm{Hz}$ appear more disperse. This period is weakly influenced by microseismic noise (see Fig. 3). This appears indeed on the frequency band $[0.1-0.3] \mathrm{Hz}$, since this is the narrow band of microseismic noise.

To continue, we claim that the tremor at Stromboli in the frequency band $[0.1-0.5] \mathrm{Hz}$ is well polarised and points to the craters when the energy is relatively low. Moreover, the signals can be decomposed into independent signals that show the same polarisation properties. When the energy grows up, the oscillations produced by source processes are often hidden and the rectilinearity of the motion decreases. But a direction of azimuths pointing towards southeast can be isolated, probably linked to the blowing of the Scirocco wind, that influences above all the flank of the volcano directly exposed. 

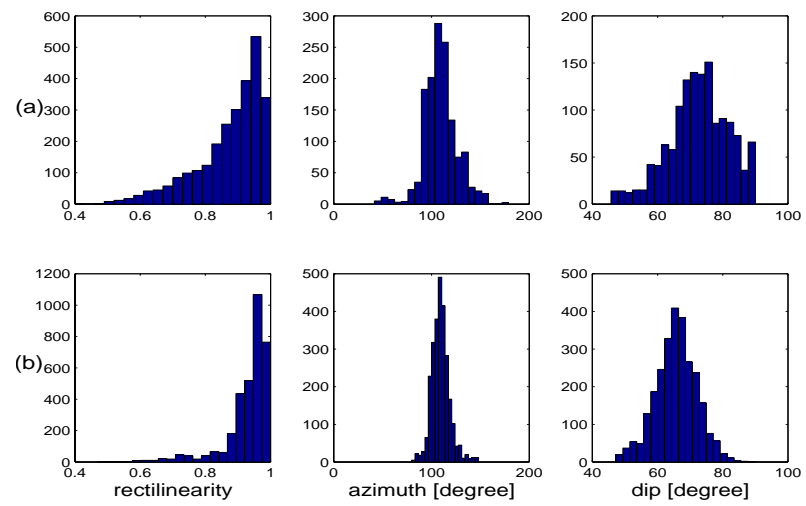

Fig. 9. Distribution of parameters of the polarisation obtained by analyzing the radially polarised tremor (19 September at 00.33 at T4) in the two sub-bands estimated: (a) $[0.1-0.3] \mathrm{Hz}$; (b) [0.3-0.5] Hz. Number of windows are on the y-axes.

\section{Zero lag cross correlation analysis}

The estimate of the source position of seismic events is usually carried out by comparing the times of the onsets on seismograms and the distances between the stations. The lack of clear arrivals in the volcanic tremor makes this procedure unapplicable. It is possible to localize the source of the tremor by using the cross-correlation function. The Zero Lag Cross Correlation (ZLCC) method is based on this idea. The classical ZLCC method has been largely used in volcanoseismology: it is based on the approximation of a planar wave front. Fixing the incoming direction of the wavefield, theoretical time delays can be estimated. They will be used to shift the seismic series recorded at the various stations. The estimated parameters of the source will be the ones which maximize the correlation between the shifted signals. Two parameters of travelling waves are estimated: the modulus of the apparent slowness (defined as the inverse of the apparent velocity) and the direction of propagation (azimuth). De Lauro et al. (2005) have applied the ZLCC technique on VLP tremors, in order to obtain information about incoming directions and to estimate the apparent velocity of travelling waves.

It is possible to improve this method by assuming that waves propagate with a circular front (ZLCC-circular). In this case, the method will depend on an additive parameter, i.e. the epicentral distance (Almendros et al., 1999). Choosing arbitrarily an origin point, we will obtain a probable epicenter zone (individuated by an azimuth and a distance from origin) and a distribution of apparent slowness. Moreover, this improvement is needed when we are dealing with a circular array or a network, and the source is supposed to be internal to it.

We apply the ZLCC-circular method on the VLP tremor. In particular, we have chosen (as above) two tremor series representative of the two polarisation states, using the ver-
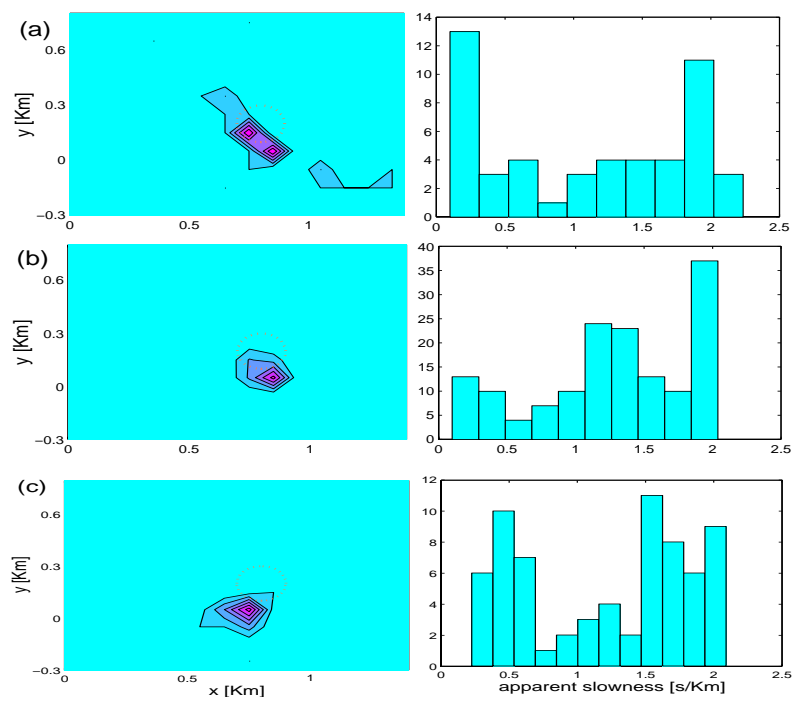

Fig. 10. Estimate of the source position for the VLP tremor (vertical channel): (a) $[0.1-0.3] \mathrm{Hz}$ (19 September at 00:33 UT); (b) [0.3-0.5] Hz (19 September at 00:33 (a)); (c) $[0.1-0.5] \mathrm{Hz}$ in the case of a nonpolarised state (24 September at 12:17 UT). The origin of the axis coincides with the M1 position. The estimated source region is the same in all cases, corresponding to the crater area (represented by the dotted circle). On the right, there are the relative estimates of the apparent slowness. The number of time windows are reported on the y-axis. These histograms show primarily two peaks: one in the range of $500-700 \mathrm{~m} / \mathrm{s}$ and another at $\sim 2 \mathrm{~km} / \mathrm{s}$.

tical component of motion recorded by seismometers in the middle ring, which can be considered planar. For the radially polarised state, we repeat the analysis for the two frequency sub-bands previously distinguished. We have used a search grid for the $\mathrm{x}$ and $\mathrm{y}$ components of slowness varying in the range $[-2: 2] \mathrm{s} / \mathrm{km}$, with a step of $0.1 \mathrm{~s} / \mathrm{km}$. The search grid for the epicentral distance from the M1 station (chosen as origin) varies from $500 \mathrm{~m}$ to $2 \mathrm{~km}$, with a step of $100 \mathrm{~m}$. The windows of the signals used for the correlation are 5 and $8 \mathrm{~s}$ long, for the two sub-bands, respectively.

In the first column of Fig. 10 we show the estimated position of the source (projected on the north-south, east-west plane) relative to the radially polarised tremor filtered in $[0.1-0.3] \mathrm{Hz}$ (Fig. 10a) and [0.3-0.5] Hz (Fig. 10b); in the second column there are the corresponding distributions of apparent slowness. The graphs are plotted using only the parameters corresponding to high values of the correlation function $(>0.9)$.

For both sub-bands, the source region appears localized near the crater area, although it is better peaked for the $0.4 \mathrm{~Hz}$ tremor. The correlation values for the tremor in the $[0.1-0.3] \mathrm{Hz}$ band are slightly lower than in the other subband, on average. Moreover, the source region appears less localized, suggesting a stronger influence of microseismic noise. 
These results confirm that both independent signals of this VLP tremor are generated into the crater area and that they are produced by vibrations of volcanic structures. In addition, the apparent velocity is estimated around $500 \mathrm{~m} / \mathrm{s}$ or a bit more for the higher frequency sub-bands, while the lower frequencies show a bimodal distribution, with an additive maximum around $2 \mathrm{~km} / \mathrm{s}$, as seen by De Lauro et al. (2005).

We have proceeded in a similar way by applying a ZLCCcircular analysis to the elliptically polarised tremor. The results are shown in Fig. 10c. We have investigated the entire band $[0.1-0.5] \mathrm{Hz}$. The position of the source appears to be approximately within the crater area, but some points do not fall into the same zone. Here, the maximum of the search grid of the epicentral distance has been extended up to $2.5 \mathrm{~km}$.

We notice that, including the windows with a correlation lower than 0.9, some points thicken on the upper border of the epicentral search grid $(2.5 \mathrm{~km})$, suggesting a sort of direct influence of an external source, as already pointed out by De Lauro et al. (2005). The apparent velocities show a large peak centered around $500-700 \mathrm{~m} / \mathrm{s}$ and another around $2 \mathrm{~km} / \mathrm{s}$, as above.

We can conclude that the ZLCC-circular analysis has furnished an estimation of the source position on the northsouth, east-west plane and of the apparent velocity of the waves. We have basically found only one source region, coincident approximately with crater area. No differences are noted for the VLP tremor with different polarisation properties nor for the two independent signals constituting the VLP tremor. The estimation of the apparent velocity has shown two maxima: one around $500-700 \mathrm{~m} / \mathrm{s}$, and another corresponding to faster waves $(\sim 2 \mathrm{~km} / \mathrm{s})$.

\section{Conclusions and discussion}

We have studied the tremor of the Stromboli volcano in the frequency range $[0.1-0.5] \mathrm{Hz}$ (VLP tremor). This band, as is well known, can be affected by microseismic noise. Despite this, De Lauro et al. (2005) have shown that it contains a coherent and radially polarised signal. In this paper, we have improved their preliminary results, by considering a large data set and a statistical approach.

Our results can be summarized and discussed as follows:

- the elliptical polarised state is consistent with the contribution coming from the external source, primarily due to the Scirocco wind. This is clearly evidenced when performing the polarisation analysis and looking at the distribution of the azimuths at the stations. On a statistical basis, the predominance of an azimuth of $120 \mathrm{deg}$ (south, southeast direction) is well evident at the stations on the east flank of the volcano. This state is always associated with more energetic signals. On the other flank, the direction pointing to the crater is also present;

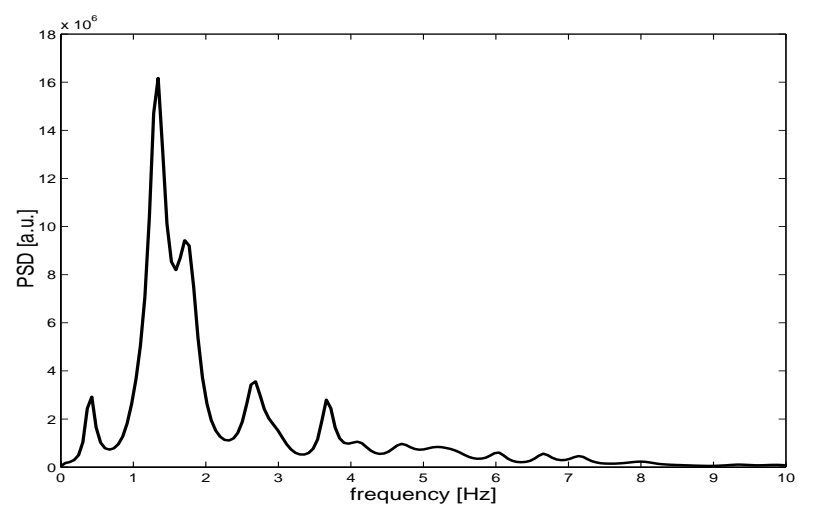

Fig. 11. Spectrum of high-frequency Strombolian tremor $(>0.1 \mathrm{~Hz})$ by using a Maximum Entropy-based algorithm.

- when the level of energy is quite low, the VLP tremor appears well polarised towards the crater area. ICA has decomposed this band into two independent sub-bands, peaked, respectively at $0.2 \mathrm{~Hz}$ and $0.4 \mathrm{~Hz}$. Though both independent source signals are still radially polarised, the first shows more dispersion. This can be ascribed to a low level of microseismic noise that prevalently influences this component peaked around $0.2 \mathrm{~Hz}$ (Webb, 1998);

- zero-lag cross-correlation technique and its modified version show that in the case of radial polarisation, the source is located in the crater area. When the energy grows and the polarisation becomes elliptical, an external source is adding. This external source, for its features of polarisation and incoming direction, can be ascribed to the blowing of the Scirocco wind.

We conclude that the two sources, wind and degassing, that impact on the vibration of the volcano, produce the same macroscopic effect. When the two sources act simultaneously (the more energetic state), the $0.2-\mathrm{Hz}$ peaked signal is affected by microseismic noise. We are observing a typical behaviour of vibrating cavities: by increasing the source energy, the fundamental peak in the spectrum shifts. Then this peak is superimposed on the $0.4-\mathrm{Hz}$ peak, making indistinguishable the two separated signals extracted in the less energetic state.

Often, people discuss the possibility of describing the high frequency signals of Stromboli as due to a suitable vibrating cavity (e.g. organ pipe model). Our results evidence a new component in the frequency band $[0.1-0.5] \mathrm{Hz}$ that supports this model.

Organ pipes are generally grouped into two classes: cylindrical and conic pipes. The first pipes have a symmetric shape and they are characterised by a decreasing exponential spectrum (the first mode is the most energetic and so on). The second pipes are asymmetrically shaped or not homogeneous (i.e. with some holes or restrictions), and they 
are characterised by a bell-shaped spectrum, in which the first mode is less energetic than the others. If we consider the cleaned spectrum of the Strombolian tremor at a high frequency $(>0.1 \mathrm{~Hz})$ computed using a Maximum Entropybased algorithm (Burg (1967)) (see Fig. 11), we recognize a spectral shape like a conic pipe (Benade and Lutgen, 1988).

We conclude that there is some evidence that the Strombolian conduit vibrates like a very complex asymmetric vibrating cavity.

Edited by: M. Thiel

Reviewed by: two referees

\section{References}

Acernese, F., Ciaramella, A., De Martino, S., Falanga, M., and Tagliaferri, R.: Neural Networks for blind sources separation of Stomboli explosion quakes, IEEE Trans. Neural Netws., 14, 167-175, 2003.

Acernese, F., Ciaramella, A., De Martino, S., Falanga, M., Godano, C., and Tagliaferri, R.: Polarisation analysis of the independent components of low frequency events at Stromboli volcano (Eolian Island, Italy), J. Volc. Geotherm. Res., Special Issue: an introduction, edited by: Harris, A., Hort, M., Ripepe, M., 137, 1-3, 153-168, doi:10.1016/j.jvolgeores.2004.05.005, 2004.

Almendros, J., Ibanez, J. M., Alguacil, G., and Del Pezzo, E.: Array analysis using circular wavefront geometry: an application to locate the nearby seismo-volcanic source, Geophys. J. Int., 136, 159-170, 1999.

Bell, A. J. and Sejnowski, T. J.: An information-maximisation approach to blind separation and blind deconvolution, Neural Comp., 7, 1129-1159, 1995.

Benade, A. H. and Lutgen, S. J.: The saxophone spectrum, J. Acoust. Soc. Am., 83, 1900-1907, 1988.

Bishop, M. C.: Neural Networks for Pattern Recognition, Clarendon Press-Oxford, 1995.

Bottiglieri, M., De Martino, S., Falanga, M., Godano, C., and Palo, M.: Statistics of inter-time of Strombolian explosion-quakes, Europhys. Lett., 72, 3, 492-498, doi:10.1209/epl/i2005-10258-0, 2005 and Research Highlights, Nature Physics, 1, December, 2005.
Burg, J. P.: Maximum entropy spectral analysis, in: Modern Spectral Analysis, edited by: Childers, D. G., IEEE Press, New York 90, 1881-1893, 1967.

Chouet, B. A., Saccorotti, G., Martini, M., Dawson, P., De Luca, G., Milana, G., and Scarpa, R.: Source and path effect in wavefield of tremor and explosions at Stromboli Volcano, Italy, J. Geophys. Res., 102, 15 129-15 150, 1997.

Chouet, B. A., Dawson, P., De Luca, G., Martini, M., Milana, G., Saccorotti, G., and Scarpa, R.: Array analyses of seismic wavefields radiated by eruptive activity at Stromboli volcano, Italy, CNR-Gruppo Nazionale per la Vulcanologia, Felici editore, Ospedaletto, Pisa, 1998.

Chouet, B. A., Saccorotti, G., Dawson, M., Martini, M., Scarpa, R., De Luca, G., Milana, G., and Cattaneo, M.: Broadband measurements of sources of explosions at Stromboli Volcano, Italy, Geophys. Res. Lett., 26, 13, 1937-1940, 1999.

Chouet, B. A., Dawson, P., Ohminato, T., Martini, M., Saccorotti, G., Giudicepietro, F., De Luca, G., Milana G., and Scarpa, R.: Source mechanism of explosions at Stromboli determined from moment tensor inversions of very-long-period data, J. Geophys. Res., 108, B1, 2019, doi:10.1029/2002JB001919, 2003.

Ciaramella, A., De Lauro, E., De Martino, S., Di Lieto, B., Falanga, M., Tagliaferri, R.: Characterization of Strombolian events by using Independent Component Analysis, Nonlin. Proc. Geophys., 11, 453-461, 2004.

De Lauro, E., De Martino, S., Falanga, M., Palo, M., and Scarpa, R.: Evidence of VLP volcanic tremor in the band [0.2-0.5] Hz at Stromboli Volcano, Italy, Geophys. Res. Lett., 32, L17303, doi:10.1029/2005GL023466, 2005.

Del Pezzo, E., Godano, C., Gorini, A., and Martini, M.: Wave polarization and location of the source of the explosion quakes at Stromboli volcano, IAVCEI Proceed. in Volcanology III, in: Volcanic seismology, edited by: Gasparini, P., Scarpa, R. and Aki, K., Springer Verlag, Heidelberg, 279-296, 1992.

De Martino, S., Falanga, M., Scarpa, R., and Godano, C.: Very long period volcanic tremor at Stromboli, Italy, Bulletin of Seismological Society of America, 95, 3, 1186-1192, 2005.

Hyvärinen, A., Karhunen, J., and Oja, E.: Independent Component Analysis, John Wiley \& Sons, New York, 2001.

Kanasewich, E. R.: Time sequence analysis in geophysics, Univ. of Alberta Press, Edmonton-Canada, 1981.

Kostelich, E. J. and Schreiber, T.: Noise reduction in chaotic time series data: A survey of common methods, Phys. Rev. E, 48, 1752-1763, 1993.

Webb, S. C.: Broadband seismology and noise under the ocean, Rev. Geophys., 36, 105-142, 1998. 